\title{
ORIGINAL
}

\section{PUBLICACIONES SOBRE MUJERES, SALUD Y GÉNERO EN ESPAÑA (1990-2005)}

\section{Esther Castaño-López, Juncal Plazaola-Castaño, Julia Bolívar-Muñoz, Isabel Ruiz-Pérez} Escuela Andaluza de Salud Pública. Granada.

\section{RESUMEN}

Fundamento: Los estudios sobre mujeres, género y salud son cada vez más frecuentes en España. El objetivo de esta investigación ha sido conocer los temas tratados en estas publicaciones, las disciplinas que los estudian, el sexo de la persona que firma en primer lugar y si han sido abordadas con enfoque de género.

Métodos: Se llevó a cabo una búsqueda bibliográfica en bases de datos de Biomedicina, Sociología, Enfermería, Antropología y Psicología. Las principales palabras clave empleadas fueron [mujeres o género] y [salud] y [España]. Se incluyeron artículos originales, revisiones y monografías, nacionales e internacionales, publicadas entre 1990 y 2005 . Las variables contempladas fueron el tema de estudio, área de conocimiento, enfoque de género y sexo de la primera autoría. Se incluyeron 298 trabajos.

Resultados: Los temas más abordados son salud sexual y reproductiva $(39,2 \%$ ) y salud mental $(12,4 \%)$. El $58,7 \%$ de los trabajos tienen como primera autora a una mujer y el $47,3 \%$ no tienen enfoque de género. El 85,3\% de las publicaciones sobre salud sexual y reproductiva no incorporan la perspectiva de género, mientras el $94,1 \%$ de los trabajos sobre trabajo productivo y reproductivo sí. El 80,4\% de los trabajos en medicina no tienen enfoque de género.

Conclusiones: Los trabajos con enfoque de género están firmados mayoritariamente por mujeres como primeras autoras, si bien son escasos. En el material estudiado, la salud de las mujeres sigue circunscribiéndose a la reproducción, siendo necesario incorporar otros determinantes de salud.

Palabras clave: Mujeres. Salud de las mujeres. Feminismo. Ciencias Sociales. España.

Correspondencia:

Esther Castaño López

Escuela Andaluza de Salud Pública

Campus Universitario de Cartuja

Cuesta del Observatorio 4

Apartado 2070

18080 Granada.

Correo electrónico: esther.castano.easp@juntadeandalucia.es

\section{ABSTRACT \\ Publications on Women, Health and Gender in Spain (1990-2005)}

Background: The studies on women, gender and health are increasingly more frequent in Spain. This research is aimed at ascertaining the topics dealt with in these publications, the disciplines studying said topics, the sex of the leading author and whether these topics have been approached from a gender-related standpoint.

Methods: A search was conducted for published studies in Biomedicine, Sociology, Nursing, Anthropology and Psychology databases. The main key words used were [women or gender] and [health] and [Spain]. Original articles, reviews and monographic studies, both national and international, published within the 1990-2005 period were included. The variables taken into consideration were the topic of study, field of expertise, gender focus and sex of those publishing the work. A total of 298 works were included.

Results: The topics dealt with the most were sexual and reproductive health $(39.2 \%)$ and mental health (12.4\%). A total of $58.7 \%$ of the works were authored by a woman as the leading author and $47.3 \%$ had no gender focus. A total of $85.3 \%$ of the publications on sexual and reproductive health do not incorporate the gender standpoint, whilst $94.1 \%$ of the works on productive and reproductive work did however include this standpoint. A total of $80.4 \%$ of the works in Biomedicine have no gender focus.

Conclusions: Even though they are scarce, the works having a gender focus are signed mostly by women as the leading authors. In the material studied, women's health continues to be confined to reproduction, it being necessary for other factors having a direct bearing on women's health to be included.

Key words: Women. Women's health. Feminism. Social Sciences. Spain. 


\section{INTRODUCCIÓN}

Los estudios sobre mujeres, género y salud son cada vez más frecuentes en nuestro país. Por ello, cabe preguntarse qué se está investigando y publicando bajo esta denominación. Por un lado se están estudiando temas «genuinamente femeninos» como por ejemplo cáncer de útero, aborto, menopausia, etc., de modo que se trataría de trabajos sobre la salud de las mujeres. Por otro lado, se encuentran estudios en los que se analiza un problema de salud o un hábito y los factores asociados al mismo de manera separada para mujeres y varones. En ambos casos el sexo es una variable necesaria pero no suficiente para incorporar una perspectiva de género $^{1-3}$, ya que podrían estar obviándose los diferentes determinantes y condicionantes de los procesos de salud y enfermedad en mujeres y varones.

El género debe entenderse como un concepto relacional. Son las relaciones de género y sus cambios dentro de diferentes contextos los que deberían centrar los análisis, así como las diferencias en las realidades e intereses dentro de los colectivos masculinos o femeninos ${ }^{4}$. Aparte de las diferencias anatómicas, hormonales o cromosómicas existentes entre mujeres y varones, las cuales pueden desembocar en diversas condiciones de salud y enfermedad, varones y mujeres tienen condiciones de existencia diferentes.

Las Ciencias de la Salud han abordado en mayor medida el estudio del género y la salud, aunque no lo han hecho de manera exclusiva. En la Antropología hay múltiples antecedentes de los estudios de género ${ }^{5}$, y ya en los años 50 desde la Psicología se empleaba el término género para denominar la relación entre características sexuales y psicológicas $^{2}$. Los estudios de género se desarrollaron en gran medida de la mano de las Ciencias Sociales a partir de las luchas feministas y después de que algunas mujeres accedieran a una mayor educación y, en algunos casos, a mayor igualdad. Ello fraguó las pre-condiciones necesarias para dar paso a una nueva conciencia académica más crítica con el androcentrismo dominante en la ciencia ${ }^{6}$.

Si bien la salud y el género se abordan desde diversas disciplinas, existe una importante desigualdad de género en la producción científica y publicaciones, recientemente evidenciada en la literatura ${ }^{7,8}$. El número de investigadoras en nuestro país, y el número de publicaciones con mujeres como primeras autoras han aumentado considerablemente, aunque es escaso su liderazgo en el sistema de investigación científica, desarrollo tecnológico e innovación ${ }^{9,10}$. El interés por la interacción entre género y ciencia, así como la relevancia del concepto de género en el análisis de la actividad científica, surge de investigaciones que analizan la escasez de mujeres visibilizadas a lo largo de la historia de la ciencia y las barreras que obstaculizan el acceso de las mujeres al conocimiento y la tecnología ${ }^{11-14}$.

Una de las dificultades para conocer estas desigualdades es la obtención de indicadores bibliométricos desagregados por sexo, ya que las publicaciones citan la autoría de los textos referenciados por apellido e inicial, de tal modo que resulta imposible identificar si se trata de varones o mujeres ${ }^{10}$. Un estudio realizado en nuestro país evidencia desigualdades en la primera autoría en las publicaciones de artículos científicos en el campo de la medicina y la salud pública. De los 413 artículos que revisan, sólo un $29.3 \%$ tienen como primer firmante a una mujer y además detectan diferencias en los temas tratados por ellas y ellos ${ }^{15}$.

El objetivo de esta investigación es conocer los temas tratados en las publicaciones relacionadas con la salud y las mujeres en España, las disciplinas que los estudian, el sexo de la primera autoría y si han sido abordadas con enfoque de género. 


\section{MATERIAL Y MÉTODOS}

\section{Bases de datos}

Durante los meses de marzo y abril de 2005 se llevó a cabo una búsqueda bibliográfica. Las bases de datos consultadas fueron PubMed, MedicLatina, SciELO, BDIE (Base de Datos para la Investigación en Enfermería en España), IBECS (Índice Bibliográfico Español de Ciencias de la Salud), Cuiden e ISOC (Base de Datos de Revistas de Ciencias Sociales y Humanidades). También fueron consultadas MEDLINE, Serfile, PsycINFO, Cab Health, FRANCIS, Wilson Humanities Full Text, ISD (International Science Database) y NTIS (National Technical Information Service), a partir del servidor de bases de datos SilverPlatter. Se escogieron estas bases con el objetivo de incluir las disciplinas o áreas de conocimiento que tradicionalmente han estudiado la salud de las mujeres (biomedicina, sociología, enfermería, antropología y psicología). Además, fueron seleccionadas por ser las que abarcan un mayor espectro de publicaciones y porque recogen las revistas científicas de mayor visibilidad tanto en el ámbito nacional como internacional.

\section{Estrategia de búsqueda}

La estrategia de búsqueda utilizada fue adaptada a cada una de las bases de datos consultadas. En algunas bases se utilizaron descriptores o vocabulario controlado, por ejemplo los términos MeSH (Medical Subject Headings) en Medline. En las demás bases de datos se utilizaron palabras clave $o$ vocabulario no controlado. Los descriptores y palabras clave empleados en cada base de datos se presentan en la tabla 1. En las bases que lo permitían, la búsqueda se limitó al periodo comprendido entre enero de $1990 \mathrm{y}$ abril de 2005, y en aquellas que no lo permitían se eliminaron las publicaciones anteriores a 1990 y las posteriores a abril de 2005 . Se incluyeron todos los artículos originales, revisiones y monografías que plantearan, entre sus principales objetivos, el estudio de aspectos relacionados con la salud de las mujeres en nuestro país. Se excluyeron las cartas al director, los editoriales y las comunicaciones a congresos. Dado que algunas publicaciones se encuentran en más de una base de datos, y que en una misma base de datos diferentes estrategias de búsqueda produjeron resultados solapados, las publicaciones duplicadas se tuvieron en cuenta una única vez. La búsqueda produjo un total de

Tabla 1

Descriptores y palabras clave utilizadas en cada base de datos

\begin{tabular}{|l|l|}
\hline Base de datos & Descriptores o palabras clave \\
\hline PubMed & [Spain] and [Women's health]; Spain and Women's health \\
\hline MedicLatina & $\begin{array}{l}\text { Mujer y Salud y España; Mujer and Salud and España; Mujer and España; Mujeres and España; Women } \\
\text { and Spain; Género y España; Género and Salud and España; Género and Salud }\end{array}$ \\
\hline SciELO & Gender and Health and Spain \\
\hline ISOC & Mujer o Género y Salud \\
\hline SilverPlatter* & Mujer and Salud and Spain; Género and Salud and Spain \\
\hline BDIE & Mujeres and España and Salud; Género and España \\
\hline IBECS & Mujeres and España and Salud; Género and España \\
\hline Cuiden & Mujer \& Salud; Género \& Salud; Mujer \& Salud \& España \\
\hline
\end{tabular}

[ ] Vocabulario controlado

* Las bases de datos consultadas en el servidos de bases SilverPlatter fueron: MEDLINE, Serfile, PsycINFO, Cab Health, FRANCIS, Wilson Humanities Full Text, ISD y NTIS. 
754 trabajos, de los cuales se excluyeron 456 por no cumplir los criterios de inclusión o estar duplicados. Finalmente se incluyeron en el análisis 298 trabajos.

\section{Variables contempladas}

Cada una de las publicaciones recuperadas fue clasificada según: tema de estudio:

(1) cáncer;

(2) salud sexual y reproductiva (planificación familiar, salud maternoinfantil, climaterio, menstruación, sexualidad);

(3) violencia contra las mujeres (violencia doméstica, abusos sexuales);

(4) salud mental (trastornos de la conducta alimentaria y de la imagen corporal, suicidio, depresión, ansiedad);

(5) enfermedades de transmisión sexual;

(6) trabajo productivo y reproductivo (conciliación de la vida laboral, familiar y personal, cuidados informales, salud laboral, condiciones de empleo);

(7) estilos de vida (alimentación, ejercicio físico, tabaquismo, uso y abuso de sustancias);

(8) mujeres en las profesiones sanitarias (feminización de determinadas profesiones, historia de las mujeres en la ciencia);

(9) morbilidad diferencial (diferencias en la forma de enfermar de mujeres y varones);

(10) sesgos de género en la atención socio-sanitaria;

(11) otros (inmigración, envejecimiento, pobreza, prostitución y educación sanitaria).

El tema de estudio de cada uno de los trabajos fue definido en base al título y al con- tenido del trabajo. En algunos casos fue posible acceder al texto completo, y en otras ocasiones únicamente al resumen o al título. En primer lugar se seleccionaron 20 trabajos de forma aleatoria que fueron revisados conjuntamente por las autoras, identificando dificultades a la hora de determinar el tema de estudio y unificando criterios de clasificación. Posteriormente los trabajos fueron analizados individualmente. En aquellos textos que presentaron dificultades el tema de estudio fue consensuado en grupo.

Disciplina o área de conocimiento: (1) antropología; (2) enfermería; (3) medicina; (4) psicología; (5) salud pública; (6) sociología. Este trabajo ha sido llevado a cabo desde el ámbito de la Salud Pública, y por ello se consideró de interés tratarla como un área de conocimiento específica independiente de la Medicina.

La disciplina desde la cual se llevaron a cabo cada uno de los trabajos fue definida en función del área de conocimiento de la primera autora o primer autor de los mismos, que fue definida en base a su lugar de trabajo. Así, si ésta o éste pertenecía al Departamento de Salud Pública de la Universidad X, la disciplina asignada al trabajo era salud pública y si pertenecía al Servicio de Ginecología del Hospital Y, la disciplina asignada era medicina. En los casos en que no fue posible identificar a la primera autora o primer autor, la asignación de la disciplina se hizo en base al contenido del artículo.

Enfoque de género: (1) sí; (2) no; (3) no sabe.

Para definir si un trabajo tenía enfoque de género se examinaron, a partir del resumen del mismo, los objetivos, la estrategia de análisis y la interpretación de los resultados. En algunos casos no se tuvo acceso al resumen y por lo tanto no pudo definirse esta variable $(\mathrm{N}=62)$. Se estableció que un trabajo tenía enfoque de género si, en el planteamiento de los objetivos, análisis e interpretación de la 
información, consideraba la construcción social del hecho biológico de ser mujer o varón y las diferencias y desigualdades que ésta conlleva ${ }^{16}$. Así, los datos desagregados por sexo fueron contemplados como una condición necesaria en los trabajos que incluían a mujeres y varones, aunque no suficiente para la introducción de esta perspectiva.

Sexo de la primera autoría: (1) mujer; (2) varón; (3) no sabe.

Esta clasificación se hizo en base al nombre de la primera autora o el primer autor cuando éste se conocía $(\mathrm{N}=260)$. En la mayoría de los casos fue necesario acudir a buscadores como Google para poder identificar el nombre de estas autoras y autores.

Las variables «tema de estudio» y «disciplina o área de conocimiento» fueron creadas «ad hoc» en función de los resultados.

\section{Análisis estadístico}

El análisis de los datos se realizó con el programa estadístico SPSS versión 12.0. En primer lugar se realizó un análisis descriptivo de cada una de las variables estudiadas, utilizando tablas de frecuencia (número de casos y porcentaje). Posteriormente se crearon tablas de contingencia para estudiar la distribución de cada una de las variables en relación con las demás.

\section{RESULTADOS}

Como se muestra en la tabla 2, los temas más abordados por los trabajos de investigación localizados sobre mujer y salud, realizados en nuestro país y publicados en los últimos 15 años, son la salud sexual y reproductiva $(39,2 \%)$ y la salud mental $(12,4 \%)$. Los temas menos abordados son las enfermedades de transmisión sexual $(2,3 \%)$ y los estilos de vida $(4,4 \%)$.

En cuanto a las áreas de conocimiento que estudian la salud de las mujeres, el 36,9\% de los artículos son de medicina y el 30,5\% de salud pública. El 3,0\% de los textos encontrados son sociológicos y el 1,3\% se corresponde con artículos de antropología.

Se desconoce el sexo de la autoría en el $12,8 \%$ de los estudios analizados. En los trabajos en los que se tuvo acceso a este dato un alto porcentaje de primeras autoras fueron mujeres $(58,7 \%)$ frente al $28,5 \%$ de varones.

Además, cabe destacar que se ha podido determinar el enfoque de la mayor parte de los manuscritos, incorporando perspectiva de género el $31,9 \%$ de los mismos frente al $47,3 \%$ que no la incorpora (tabla 2 ).

En la tabla 3 se puede apreciar que la salud sexual y reproductiva es objeto de estudio en la mayor parte de los trabajos localizados, tanto en aquellos cuya primera autora es una mujer $(30,3 \%)$ como en los que el primer autor es un varón $(42,4 \%)$. No obstante, el resto de trabajos firmados bien por mujeres bien por varones tienen un objeto de estudio diferente. Así, mientras las mujeres abordan con mayor frecuencia las profesiones sanitarias $(10,3 \%)$, el trabajo productivo y reproductivo $(9,7 \%)$ y la salud mental $(9,7 \%)$, sólo el 3,5\%, el 4,7\% y 12,9\% de las investigaciones realizadas por hombres abordan estos temas, respectivamente.

La totalidad de los trabajos identificados en este estudio cuyo tema de investigación son los estilos de vida carece de enfoque de género, así como el 93,3\% de los trabajos cuyo tema de investigación es el cáncer. Algo semejante sucede con la salud sexual y reproductiva, pues el 85,3\% de los artículos encontrados sobre este tema no incorpora la perspectiva de género. Por el contrario el $94,1 \%$ de los artículos que abordan la relación entre trabajo productivo y reproductivo lo hacen con enfoque de género. Igualmente, la mayoría de los trabajos sobre profesiones sanitarias $(88,2 \%)$ y sesgos de género en la atención sociosanitaria $(68,8 \%)$ parten de esta perspectiva (tabla 4). 
Tabla 2

Tema de estudio, disciplina, sexo de la primera autoría y enfoque de género en las investigaciones localizadas

\begin{tabular}{|c|c|c|}
\hline & $\mathbf{N}$ & $\%$ \\
\hline \multicolumn{3}{|l|}{ Tema de estudio } \\
\hline Cáncer & 19 & 6,4 \\
\hline Salud sexual y reproductiva & 98 & 39,2 \\
\hline Violencia contra las mujeres & 15 & 5,0 \\
\hline Salud mental & 37 & 12,4 \\
\hline Enfermedades de transmisión sexual & 7 & 2,3 \\
\hline Trabajo productivo y reproductivo & 24 & 8,1 \\
\hline Estilos de vida & 13 & 4,4 \\
\hline Mujeres en las profesiones sanitarias & 23 & 7,7 \\
\hline Morbilidad diferencial & 25 & 8,4 \\
\hline Sesgos de género en la atención sociosanitaria & 16 & 5,4 \\
\hline Otros & 21 & 7,0 \\
\hline \multicolumn{3}{|l|}{ Disciplina } \\
\hline Antropología & 4 & 1,3 \\
\hline Enfermería & 55 & 18,5 \\
\hline Medicina & 110 & 36,9 \\
\hline Psicología & 29 & 9,7 \\
\hline Salud Pública & 91 & 30,5 \\
\hline Sociología & 9 & 3,0 \\
\hline \multicolumn{3}{|l|}{ Sexo de la primera autoría } \\
\hline Mujer & 175 & 58,7 \\
\hline Varón & 85 & 28,5 \\
\hline Perdidos & 38 & 12,8 \\
\hline \multicolumn{3}{|l|}{ Enfoque de género } \\
\hline Sí & 95 & 31,9 \\
\hline No & 141 & 47,3 \\
\hline Perdidos & 62 & 20,8 \\
\hline TOTAL & 298 & 100 \\
\hline
\end{tabular}

El $80,4 \%$ de los trabajos de medicina no tiene enfoque de género, así como el 74,1\% de los de psicología y el $62,5 \%$ de los de enfermería. Por otra parte, el $67,4 \%$ de los textos encontrados de salud pública sí incorpora este enfoque (figura 1 ).
La figura 2 muestra la distribución del enfoque de género en relación al sexo del primer autor o autora. El 82,6\% de las investigaciones cuya primera autora es una mujer y el $53,7 \%$ de los trabajos firmados por varones tienen enfoque de género. 
Tabla 3

Distribución del sexo de la primera autoría en relación al tema de estudio*

\begin{tabular}{|l|c|c|}
\hline & \multicolumn{2}{|c|}{ Sexo de la primera autoría } \\
\hline Tema de estudio & $\begin{array}{c}\text { Mujer } \\
\text { N (\%) }\end{array}$ & $\begin{array}{c}\text { Varón } \\
\text { N (\%) }\end{array}$ \\
\hline Cáncer & $12(6,9)$ & $5(5,9)$ \\
\hline Salud sexual y reproductiva & $53(30,3)$ & $36(42,4)$ \\
\hline Violencia contra las mujeres & $11(6,3)$ & $4(4,7)$ \\
\hline Salud mental & $17(9,7)$ & $11(12,9)$ \\
\hline Enfermedades de transmisión sexual & $6(3,4)$ & $0(0,0)$ \\
\hline Trabajo productivo y reproductivo & $17(9,7)$ & $4(4,7)$ \\
\hline Estilos de vida & $8(4,6)$ & $3(3,5)$ \\
\hline Mujeres en las profesiones sanitarias & $18(10,3)$ & $3(3,5)$ \\
\hline Morbilidad diferencial & $11(6,3)$ & $8(9,4)$ \\
\hline Sesgos de género en la atención sociosanitaria & $10(5,7)$ & $6(7,1)$ \\
\hline Otros & $12(6,9)$ & $5(5,9)$ \\
\hline TOTAL & $175(67,3)$ & $85(32,7)$ \\
\hline
\end{tabular}

* No fue posible identificar el sexo de los primeros autores o autoras de 38 investigaciones N:260.

\section{Figura 1}

Distribución del enfoque de género en relación a la disciplina de estudio

$\square$ Con enfoque
$\square$ S in enfoque

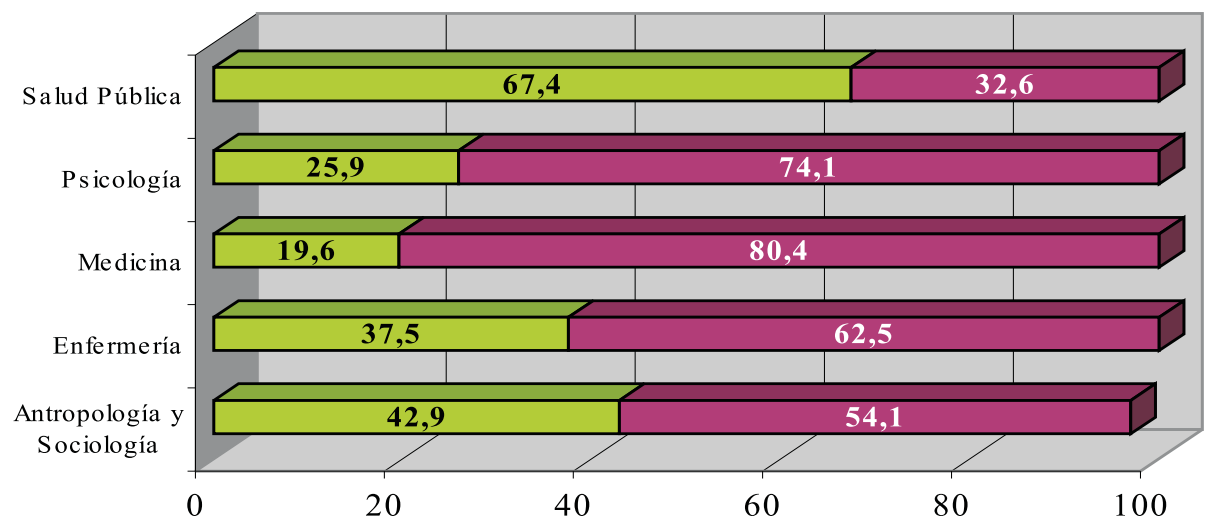

$\mathrm{N}=236$ 
Tabla 4

Distribución del enfoque de género en relación al tema de estudio*

\begin{tabular}{|l|c|c|}
\hline & \multicolumn{2}{c|}{ Enfoque de género } \\
\hline Tema de estudio & $\begin{array}{c}\text { Sí } \\
\text { N (\%) }\end{array}$ & $\begin{array}{c}\text { No } \\
\text { N (\%) }\end{array}$ \\
\hline Cáncer & $1(6,7)$ & $14(93,3)$ \\
\hline Salud sexual y reproductiva & $10(14,7)$ & $58(85,3)$ \\
\hline Violencia contra las mujeres & $7(58,3)$ & $5(41,7)$ \\
\hline Salud mental & $13(40,6)$ & $19(59,4)$ \\
\hline Enfermedades de transmisión sexual & $4(80,0)$ & $1(20,0)$ \\
\hline Trabajo productivo y reproductivo & $16(94,1)$ & $1(5,9)$ \\
\hline Estilos de vida & $0(0)$ & $13(100)$ \\
\hline Mujeres en las profesiones sanitarias & $15(88,2)$ & $2(11,8)$ \\
\hline Morbilidad diferencial & $9(39,1)$ & $14(60,9)$ \\
\hline Sesgos de género en la atención sociosanitaria & $11(68,8)$ & $5(31,3)$ \\
\hline Otros & $9(50,0)$ & $9(50,0)$ \\
\hline TOTAL & $95(40,3)$ & $141(59,7)$ \\
\hline
\end{tabular}

* No fue posible identificar el enfoque en 62 investigaciones N:236.

Figura 2

Distribución del enfoque de género en relación al sexo de la primera autoría

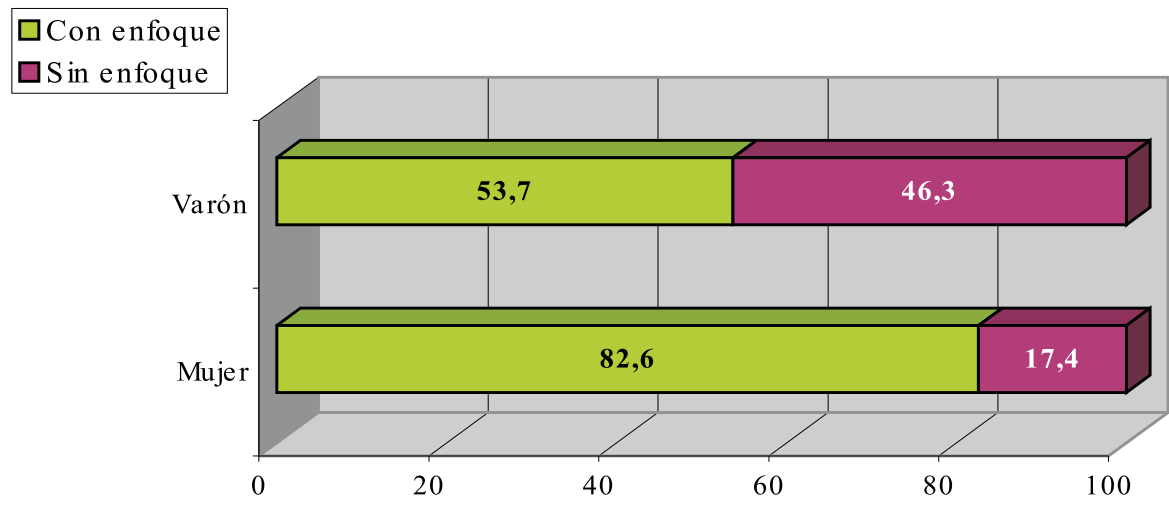

$\mathrm{N}=209$ 
Atendiendo a los grandes temas que abordan las diferentes disciplinas, cabe destacar que tanto en medicina como en enfermería y salud pública se publican, principalmente, estudios sobre salud sexual y reproductiva $(60,0 \%, 40,9 \%$ y $17,6 \%$ respectivamente). En psicología la salud mental es el tema que mayor número de trabajos acapara $(55,2 \%)$. Desde la antropología y la sociología el tema que más se aborda es el trabajo productivo y reproductivo $(46,2 \%)$.

\section{DISCUSIÓN}

Los datos muestran que la salud sexual y reproductiva es el tema que más publicaciones acapara, y que la mayoría de estos estudios no incorpora el enfoque de género a sus análisis. Se trata de unos resultados relevantes, a la vez que totalmente previsibles si tenemos en cuenta que la salud sexual y reproductiva de las mujeres es patrimonio casi exclusivo de la Ginecología, disciplina todavía hoy practicada fundamentalmente por varones y carente, en sus orígenes, de enfoque de género ${ }^{17,18}$. Desde su profesionalización se erigió como la única encargada de velar por las salud de las mujeres, legitimando su especialidad y conocimientos mediante un exacerbado corporativismo que apartó del ejercicio de la profesión a las mujeres que tradicionalmente se habían encargado de las salud reproductiva femenina: las matronas ${ }^{19}$.

La forma en que el cuerpo de las mujeres fue estudiado y definido por los ginecólogos durante la primera mitad del siglo XX también revela muchas cuestiones acerca del originario androcentrismo de la ciencia médica ${ }^{20-24}$. Así lo muestra la prescripción de fármacos psicótropos a mujeres, en la mayoría de los casos para la enfermedad que no tiene nombre, esto es, la medicalización de ciclos vitales como la menopausia ${ }^{25}$ y el malestar emocional de las mujeres ${ }^{26}$.
¿Qué pasa, entonces, con los demás factores que ponen en peligro nuestra salud?. El caso de las enfermedades cardiovasculares es un buen ejemplo para denunciar el olvido de la salud de las mujeres en ciertos ámbitos. Aunque la cardiopatía isquémica es la primera causa de muerte tanto en mujeres como en varones, se ha constatado una menor supervivencia de éstas a un fallo cardiaco ${ }^{27}$. Igualmente, llama la atención que a pesar de ser un tema de relevante actualidad política y social en nuestro país y de constituir un problema de Salud Pública de primer orden, sólo el 5\% de los trabajos publicados tienen como objeto de estudio la violencia contra las mujeres. Quizás el contexto de intimidad en el que se produce, los tabúes asociados a esta violencia, así como la ausencia de consenso en su definición puedan explicar la escasez de trabajos al respecto.

Como limitación de este trabajo encontramos que, aunque se ha incluido un importante número de documentos que ha permitido obtener una amplia visión general del problema de estudio, no se ha podido profundizar en la información recogida puesto que se trata de una revisión de la literatura a partir de los resúmenes de los trabajos localizados. Esto se ha evidenciado en las dificultades encontradas a la hora de clasificar los artículos analizados según incorporen o no una perspectiva de género. Los estudios de género están de moda lo cual, a pesar de las innumerables ventajas, supone algunos inconvenientes. Son numerosas las veces que se emplea la palabra género cuando, en realidad, se habla de la variable sexo o de mujer. Los títulos de algunos trabajos auguran un análisis de género, sin embargo al leer los resúmenes de los mismos se comprueba que no es así. En este sentido, es posible que esa falta de sintonía entre títulos y resúmenes se produzca también entre resúmenes y textos, simulando los primeros un supuesto análisis de género que luego no se encuentra en el cuerpo de los trabajos.

Por otra parte, se han incorporado al estudio bases de datos propias de las Ciencias 
Sociales, lo cual incrementa la potencia de este trabajo. A partir de la segunda mitad del siglo XX fueron las Ciencias Sociales las catalizadoras de los movimientos feministas, introduciendo la perspectiva de género en las academias. Así por ejemplo, Betty Friedman o Simone de Beauvoir, destacadas militantes feministas y también científicas, denunciaron la subordinación de las mujeres en el pensamiento occidental ${ }^{28,29}$. Con el planteamiento de este trabajo se ha pretendido incorporar esta importante trayectoria de investigación feminista.

La investigación se ha limitado al estudio de publicaciones incluidas en un número determinado de bases de datos, evitando la tentación de asumir que el total de lo que se publica coincide plenamente con lo que se investiga. De esta forma, no ha sido objeto de estudio la literatura gris (muy habitual entre los estudios de las mujeres) ${ }^{30}$, tesis doctorales, informes de organismos públicos, etc., no visibles a través de los canales de difusión del conocimiento científico oficiales y tradicionales (bases de datos y revistas científicas de impacto).

Además, existen sesgos de publicación en tanto que los estudios de género pueden constituir un material controvertido para el mundo editorial, motivo por el cual algunos son apartados del pensamiento oficial y relegados a canales de difusión científica de segunda. Conviene recordar también que las mujeres encuentran más dificultades para publicar sencillamente porque tropiezan con muchas más trabas que los varones para hacer carrera en el mundo de la ciencia ${ }^{10,31}$ (como en todos los demás ámbitos de poder $)^{32,33}$. La historia del acceso de las mujeres al conocimiento así lo muestra: el papel de las mujeres en determinada actividad es inversamente proporcional al prestigio de la misma ${ }^{10} \mathrm{Y}$ es que todos los mecanismos para llegar al círculo de honorables, están teñidos, consciente o inconscientemente, de una cultura de la masculinidad que genera perspectivas y cosmovisiones androcéntricas ${ }^{34}$.
Si a ello añadimos, como se observa en este estudio, que son los artículos firmados por mujeres los que incorporan la perspectiva de género con más frecuencia, el sesgo de publicación que se produce en estos casos se multiplica por dos (mujer y género). Así, es preciso reflexionar sobre las consecuencias que tiene el hecho de que sean las mujeres quienes más investigan en género y salud.

Como conclusión, destacar que la salud como fenómeno multidimensional no se estudia exclusivamente desde el ámbito de la medicina y, por ello, si queremos comprender los procesos de salud-enfermedad en mujeres y varones deberíamos considerar los trabajos realizados también desde otras disciplinas, como por ejemplo la sociología o la antropología. Igualmente, y a pesar de que los estudios de género están «de moda», los resultados de este trabajo muestran que son escasos las investigaciones que realmente hacen un análisis de la salud de las personas considerando la realidad diferencial y desigual de mujeres y varones. Además, la mayoría de estos trabajos son liderados por mujeres, a pesar de su escasa visibilidad en el ámbito de la investigación en este país. Para finalizar, este trabajo vuelve a poner de manifiesto que la salud de las mujeres sigue circunscribiéndose a las funciones reproductoras y que es necesario incorporar otros determinantes, como los estilos de vida o los cuidados informales. Finalmente, subrayar de nuevo la necesidad de que las publicaciones científicas permitan identificar el sexo de sus autores y autoras.

\section{BIBLIOGRAFÍA}

1. Rohlfs I, Borrell C, do C Fonseca M. Género, desigualdades y salud pública: conocimientos y desconocimientos. Gac Sanit 2000; 14 (Suplemento 3): 60-71.

2. Castañeda I, Astraín ME, Martinez V, Sarduy C, Alfonso AC. Algunas reflexiones sobre el género. Rev Cubana Salud Pública 1999; 25(2):129-42. 
3. Naciones Unidas. Cuarta Conferencia sobre la Mujer. Beijing, 1995. Disponible en: www.cinu. org.mx/temas/mujer/confmujer.htm [citado 05 de septiembre de 2005].

4. Esteban ML. El género como categoría analítica. Revisiones y aplicaciones a la salud. Cuad Psiquiatr Comunitaria 2003; 3:22-39.

5. González L. Antropología: Los estudios de género. Lamusa digital. Disponible en: www.uclm.es/ lamusa/index.asp?lengua=es [citado 05 de enero de 2006].

6. Castro R, Bronfman M. Feminist theory and medical sociology: issues for discussion. Cad. Saúde Pública. 1993; 9(3): 375-94.

7. Scott J. Measures of sex differences in scientific productivity. Social Forces 1992; 71:159-78.

8. Wennerås $\mathrm{C}$, Wold $\mathrm{A}$. Nepotism and sexism in peer-review. Nature 1997; 387: 341-3.

9. Alcalá P, Bordons M, García de Cortázar ML, Griñón M, Guil A, Muñoz A, et al. Mujer y Ciencia. La situación de las mujeres investigadoras en el sistema español de ciencia y tecnología. Madrid: Fundación Española de Ciencia y Tecnología; 2005. Disponible en: www.fecyt.es/default.cfm?id_seccion=1115\&id_sec=1114\&nivel=1 [citado 05 de septiembre de 2005].

10. Pérez E. ¿El poder de una ilusión?: Ciencia, Género y Feminismo; 2000. Disponible en: www.campus-oei.org/salactsi/sedeno2.htmM.T. [citado 19 de octubre de 2005].

11. Barral MJ, Miqueo C, Magallón C, Sánchez MD editores. Interacciones ciencia y género. Barcelona: Icaria; 1999.

12. O'Rand A. Women in Science: Career Processes and Outcomes. Social Forces 2004;82(4):1669-71.

13. Ortiz-Gómez T, Birriel-Salcedo F. Ortega del Olmo R. Género, profesiones sanitarias y salud pública. Gac Sanit 2004;18:189-94.

14. Davo MC, Vives C, Álvarez-Dardet C. Why are women underused in the JECH peer review process? J Epidemiol Community Health. 2003;57: 936-7.

15. Schiaffino A, García M, Fernández E. Autoría y presentación de resultados según el género en cuatro revistas biomédicas españolas Gac Sanit 2001;15:251-4.
16. Esteban ML. El estudio de la salud y el género: las ventajas de un enfoque antropológico y feminista. Salud Colectiva 2006; 2(1):9-20.

17. Domínguez C, de Miguel J. La justificación médica de la desigualdad sexual: ideologías de los ginecólogos españoles sobre la mujer. Doctor, septiembre: 54-60 y octubre: 56-65.1977.

18. Domínguez C, de Miguel J. El mito de la inmaculada concepción. Barcelona: Anagrama;1979.

19. Cabré i Pairet M, Ortiz-Gómez T, (eds.) Sanadoras, matronas y médicas en Europa, siglos XII-XX. Barcelona: Icaria; 2001.

20. Scully D, Bart P. A funny thing happened on the way to the orifice: Women in Gynecology textbooks. AJS 1973; 78:1043-1050.

21. Castaño E. ¿Aportaciones? de la Ginecología al Feminismo. Letra Clara. Revista de la Facultad de Filosofía y Letras de Granada (monográfico de género) 2003; 14(15): 130-32.

22. Laqueur T. La construcción del sexo. Cuerpo y género desde los griegos hasta Freud. Madrid: Crítica; 1994.

23. Martín E. The egg and the sperm: How has science constructed a romance based on stereotipical malefemale roles. Sing 1991;16:485-500.

24. Schiebinger L. The mind has no sex?: Women in the origins of the modern science. Cambridge, Ma: Harvard University Press; 1989.

25. Ruiz I, Bermejo MJ. Conocimientos de las mujeres menopausicas respecto a la terapia hormonal sustitutiva. Gac Sanit 2004;18(1):32-7.

26. Ruiz MT, Verdú MT. Sesgo de género en el esfuerzo terapéutico. Gac Sanit 2004;18 (Supl1):118125 .

27. Marrugat J, Sala J, Masia R, Pavesi M, Sanz G, Valle $\mathrm{V}$ et al. Mortality differences between men and women following first myocardial infarction. RESCATE Investigators. Recursos Empleados en el Síndrome Coronario Agudo y Tiempo de Espera. JAMA 1998; 280(16):1405-9.

28. Friedman B. La mística de la feminidad. Madrid: Júcar; 1974.

29. Beauvoir S. El segundo sexo. Buenos Aires: Siglo XX; 1987. 
30. Torres I, Muñoz, AM. Fuentes de información para los Estudios de las Mujeres. Granada: Editorial Universidad de Granada. Colección Feminae; 2000.p.16.

31. García de León MA. La excelencia científica. Hombres y mujeres en las Reales Academias. Instituto de la mujer. Madrid: Ministerio de trabajo y asuntos sociales. Secretaria general de políticas de igualdad; 2003. Disponible en: www.mtas.es/ mujer/publicaciones/docs/excelencientif.pdf. [citado 13 de enero de 2006].
32. Chinchilla N, Poelmans S. Mujeres directivas bajo el techo de cristal. Disponible en: www.uv.es/csif/ Mujeres\%20directivas.doc [citado 9 de enero de 2006].

33. Barbera E, Ramos A, Sarrió M, Candela C. Más allá del techo de cristal. Revista del Ministerio de Trabajo y Asuntos Sociales 2002; 40:55-68.

34. Sánchez D. Androcentrismo en la ciencia. Una perspectiva del análisis crítico del discurso. En: Barral MJ., et al., (ed.) Interacciones ciencia y género. Discursos y prácticas científicas de mujeres. Barcelona: Icaria;1999: 161-84. 